\title{
Ionization-induced laser-driven QED cascade in noble gases
}

\author{
I. I. Artemenko, I. Yu. Kostyukov \\ Institute of Applied Physics, Russian Academy of Science, \\ 46 Uljanov str., 603950 Nizhny Novgorod, Russia
}

\begin{abstract}
A formula for the ionization rate in extremely intense electromagnetic field is proposed and used for numerical study of QED (quantum-electrodynamical) cascades in noble gases in the field of two counter-propagating laser pulses. It is shown that the number of the electron-positron pairs produced in the cascade increases with the atomic number of the gas where the gas density is taken to be reversely proportional to the atomic number. While the most electrons produced in the laser pulse front are expelled by the ponderomotive force from region occupied by the strong laser field there is a small portion of the electrons staying in the laser field for a long time until the instance when the laser field is strong enough for cascading. This mechanism is relevant for all gases. For high- $Z$ gases there is an additional mechanism associated with the ionization of inner shells at the the instance when the laser field is strong enough for cascading. The role of both mechanisms for cascade initiation is revealed.
\end{abstract}

PACS numbers: 12.20.-m,79.70.+q,42.50.Ct,52.27.Ep

\section{INTRODUCTION}

Recently (quantum-electrodynamical) QED cascades in a strong laser field attracts much attention [1]3. The upcoming laser facilities will be able to generate laser pulses with the total power up to $10 \mathrm{PW}$ [4, 5]. It is generally believed that such power can be sufficient to observe QED cascading in laboratory condition [6] 8 . A cascade develops as a sequence of elementary QED processes: photon emission by electrons and positrons in the laser field alternates with pair production as a result of interaction between high energy photon and laser photons (Breit-Wheeler process [9]). Such sequence leads to avalanche-like production of electron-positron plasma and gammas. The number of the cascade particles can be so great that they will affect the laser field dynamics. In particularly, the laser field can be absorbed in self-generated plasma [10, 11].

Several configurations of the laser field are proposed to minimize laser power needed for cascading. One of the most simple configuration is the superposition of two counter-propagating laser pulses. It is shown [12] that the linear polarization of laser radiation is more favorable for cascading than circular one in low intensity limit. The laser-dipole wave can provide development of QED cascade at the laser power below $10 \mathrm{PW}[8]$. The field structure which is very similar to the dipole wave can be formed by 12 laser pulses [13]. Another laser configuration providing QED cascading at power level below $10 \mathrm{PW}$ can be constructed by coherent summation of sevaral laser pulses with elliptical polarization [6]. The focal spot size has a crucial importance for QED cascading [7. On the one hand, by reducing the size of the focal spot at a given power it is possible to increase the intensity of the laser field, thereby increasing the proba-

*Electronic address: kost@appl.sci-nnov.ru bility of QED processes. On the other hand, if the spot size is not large enough the cascade particles may escape quickly from cascade volume thereby suppressing cascade development.

In the high intensity limit the cascade can be initiated by the spontaneous creation of electron-positron pairs out of vacuum (self-seeded QED cascades) 10. In the low intensity limit and near the intensity threshold, the seed particles are needed to trigger cascading. The seeded particles can be either electrons [6, 11, 12] or high energy photons 14. The electrons as a light particles can be expelled from the cascade region by the ponderomotive potential of the laser field before the field strength reaches a maximum and only a small portion of the seed electrons may survive to trigger cascade [12. Expulsion of the highly relativistic electrons by the ponderomotive force is suppressed due to the relativistic gain in the electron mass [15. Yet the use of relativistic electrons as seed particles is hindered by high cost of high energy electron accelerators. In addition this also requires focusing of the electrons to the interaction region and synchronization between the electron beam and laser pulses. The same reasons (high cost of bright gamma ray sources, focusing and synchronization of the gamma beam) may prevent the use of high energy photons as seed particles.

Gases with high- $Z$ atoms can be a source of the seed electrons. The ionization potential of the inner electrons of high- $Z$ atoms can be so large that such electrons can leave the atoms at very high laser field strength. Therefore the seed electrons can be produced by field ionization when the laser field strength peaks and is strong enough for cascading. It is demonstrated recently [7] that cascade triggering in the field of two counter-propagating may be facilitated by employing suitable high- $Z$ gases. However the simplified model for atom ionization was used and only hydrogen and oxygen are explored for gas target. The model does not take into account the probabilistic nature of ionization, dependence of the ionization probability on the shell electron parameters, sequential 
and multiple ionization of high- $Z$ atoms. As a result this model cannot provide accurate description of the ionization and the dynamics of the seed electrons. In our work QED cascading in all noble gases irradiated by counter-propagating laser pulses is studied by three dimensional particle-in-cell Monte Carlo (3D PIC-MC) simulations with more realistic approach to laser ionization. We propose a new ionization rate formula that extended the known formula for tunnel ionization [16, 17. to extremely intense field when the potential barrier are strongly suppressed.

It should be noted that the foils made from high- $Z$ material can be also used as a laser target and the source of the seeded electrons [19. However because of large target density QED cascade development can be affected by collisional processes like bremsstrahlung and electronpositron pair production as a result of photon scattering by nuclei. Here we discusses the use of rarified gases in order to neglect collisional processes.

The paper is organized as follows. The field ionization model is described in Sec. II. In Section III, the results of 3D PIC simulations of QED cascades are presented. The distribution and the spectrum of the cascade particles are calculated. Section VI contains discussion and conclusions. The contribution of collisional effects is estimated and discussed.

\section{IONIZATION MODEL}

Effect of a strong electromagnetic field on atom may lead to ionization. In the tunnel regime of ionization the atom electrons penetrate through the potential barrier formed by the atomic field and the external electric field. At low intensities the field ionization occurs in multiphoton regime. The regime of the field ionization depends on the Keldysh parameter $\gamma_{K}=a^{-1}\left(2 I_{i} / m_{e} c^{2}\right)^{1 / 2}$, where $I_{i}$ is the ionization potential of the ion, $a=$ $e E_{L} /\left(m_{e} c \omega_{L}\right)$ is the dimensionless laser field, $E_{L}$ and $\omega_{L}$ are the laser field strength and the laser frequency, respectively, $e$ and $m_{e}$ are the charge and mass of the electron, respectively, $c$ is the speed of light 20. It is generally believed that the field ionization occurs in the tunnel regime if $\gamma \lesssim 0.5$ [21]. In our simulations the electromagnetic field can be treated as a static within the code time step. The rate of ionization in the static electric field is in the tunnel regime [16 18]:

$$
\begin{aligned}
W_{T I} & =\omega_{a} \kappa^{2} C_{k l}^{2}\left(\frac{2}{F}\right)^{2 n^{*}-m-1} \\
& \times \frac{(l+m) !(2 l+1)}{2^{m} m !(l-m) !} \cdot \exp \left(-\frac{2}{3 F}\right), \\
C_{k l}^{2} & =\frac{2^{2 n^{*}}}{n^{*} \Gamma\left(n^{*}+l^{*}+1\right) \Gamma\left(n^{*}-l^{*}\right)},
\end{aligned}
$$

where $F=E /\left(\kappa^{3} E_{a}\right)$ is the normalized electric field, $n^{*}=Z / \kappa$ is the effective principal quantum number of the ion, $Z$ is the ion charge number, $\kappa^{2}=I_{i} / I_{H}, I_{H}=$ $m_{e} e^{4} /\left(2 \hbar^{2}\right) \simeq 13.59843 \mathrm{eV}$ is the ionization potential of hydrogen, $l^{*}=n^{*}-1$ is the effective angular momentum, $l$ and $m$ are the orbital and magnetic quantum numbers, respectively, $E_{a}=m_{e}^{2} e^{5} \hbar^{-4} \approx 5.14224 \cdot 10^{9} \mathrm{~V} / \mathrm{cm}$ is the atomic electric field $\omega_{a}=m_{e} e^{4} \hbar^{-3} \simeq 4.13 \cdot 10^{16} \mathrm{c}^{-1}$ is the atomic frequency, $\hbar$ is the Planck constant, $\Gamma(x)$ is the Gamma function [22]. In the limit $n^{*} \gg 1$ formula (1) reduces to the ionization rate given in Ref. 23 .

The formula (1) is valid when the unperturbed atomic energy level is much lower then the potential barrier maximum. This condition is fulfilled when the external field strength is the much less than the critical field $E \ll$ $E_{c r}=E_{a} \kappa^{4} /(16 Z)$ [24]. For hydrogen-like atoms and ions with regard to the Stark effect $E_{c r, H}=\left(2^{1 / 2}-1\right) E_{a}$ [25. We will use expression $E_{c r}=E_{a} \kappa^{4} /(16 Z)$ because $E_{c r}<E_{c r, H}$ and the ionization rate given by $W_{T I}$ strongly deviates from results of numerical simulations for $E>E_{c r}$ [26].

The barrier suppression regime of the field ionization is relevant if $E>E_{c r}$. The analytical description of this regime is difficult since the perturbation methods are no longer valid for $E \sim E_{c r}$. For example, the analytical formula derived in Ref. 27] for the field ionization rates in the barrier suppression regime does not agree with numerical time-dependent Schredinger equation (TDSE) calculations for $E>E_{c r}[28$.

Several empirical formulas based on numerical simulations have been proposed for the ionization rate in the tunnel and barrier suppression regimes [26, 28, 29]. In Ref. 28] the piecewise formula for ionization rate is proposed so that $W_{T I}$ is used for $E \leq E_{T I Q} \sim E_{c r}$ while the quadratic dependence of the rate on the field strength is assumed for $E>E_{T I Q}$

$$
W_{Q}(E)=\omega_{a} 2.4\left(E / E_{a}\right)^{2},
$$

where $E_{T I Q}$ is a threshold electric field determined by imposing $W(E)$ to be continuous $W_{T I}\left(E_{T I Q}\right)=$ $W_{Q}\left(E_{T I Q}\right)$. Other empirical formulas providing continuous transition between tunnel and barrier suppression regimes are presented in Refs. [26, 29].

The proposed formulas for the ionization rate become not accurate in the limit of the extremely high field. For example according to numerical TDSE simulations the dependence of the ionization rate on the field strength is close to linear rather than to quadratic for $E>0.4 E_{a} \gg$ $E_{c r}$ [28. The formula proposed in Ref. [26] predicts reduction of the ionization rate in the limit $E \gg E_{c r}$ that does not agree with numerical simulations [29]. However the ionization rate formula, which is valid for $E \gg E_{c r}$, is needed to analyze field ionization for laser intensity above $10^{23} \mathrm{~W} / \mathrm{cm}^{2}$ when QED cascading is possible. For example, Eq. (1) for tunnel ionization predicts that $90 \%$ ionization of He in the electric field

$$
E(t)=a \frac{m c \omega_{L}}{e} \sin \left(\omega_{L} t\right) \sin ^{2}\left(\frac{t}{T}\right)
$$


occurs when the laser field strength achieves value $E \approx$ $4 E_{c r}$ (see Fig. 1), where $0 \leq t \leq 20 \lambda / c, a=500, \omega_{L}=$ $2 \pi c / \lambda, T=40 \omega_{L} c, \lambda=1 \mu \mathrm{m}$ is the laser wavelength. The probability for He to be not ionized can be calculated as follows $P(t)=1-\exp \left\{-\int_{-\infty}^{t} W_{T I}[|E(\tau)|] d \tau\right\}$. The ionization rate given by Eq. (1) is in orders of magnitudes greater than that numerically calculated for $E \gg E_{c r}[26$, 28. Therefore the $90 \%$ ionization of He will be achieved even at higher fields than $4 E_{c r}$.

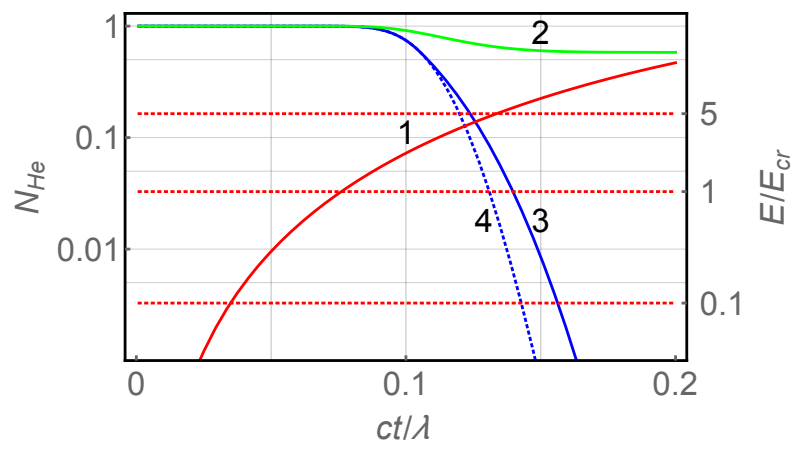

FIG. 1: The dependence of the laser field strength on time (red line 1) and the probabilities of He to be not ionized as a function of time (lines 2-4). The probabilities are calculated by integrating $W_{T I}$ (dotted blue line 4 ), $W_{P W}$ (solid blue line 3 ) and the ionization rate proposed in Ref. 26] (green line 2) over time.

In the limit of extremely strong laser field the ion field can be neglected and the electrons inside the ion can be considered as unbound just after the laser field is turned on quickly. The field of the ion with charge $Z$ at the position of the outer electron can be estimated as $E_{i}\left(r_{0}\right) \simeq e Z / r_{0}^{2}=16 E_{c r}$ at the beginning of ionization, where $r_{0} \simeq a_{B} Z / \kappa^{2}=4 r_{m}\left(E_{c r}\right)$ is the orbit radius of the electron with ionization potential $I_{i}, r_{m}(E)=(e Z / E)^{1 / 2}$ is the position of the potential barrier maximum for the electron in ion field and the external electric field $E$ and $a_{B}=\hbar^{2} /\left(m_{e} c^{2}\right)$ is the Bohr radius. Therefore the ion field can be neglected if $E \gg 16 E_{c r}$. However $16 E_{c r}$ is the maximal value of the ion field that electron feels during ionization. The condition for neglecting of the ion field can be taken as $E \gg E_{c r}$ because in this limit the ionization energy is much higher than the potential barrier maximum.

The ionization time can be estimated from model of a free electron as the time needed to accelerate the electron from the energy of the atomic level $\varepsilon_{e}=-I_{i}$ to the continuum $\varepsilon_{e}=0$ so that $I_{i}=m_{e} c^{2}\left[\left(1+a^{2} \omega_{L}^{2} \tau_{i}^{2}\right)^{1 / 2}-1\right]$, where $\varepsilon_{e}$ is the electron energy, $\tau_{i}$ is the ionization time and $a m c \omega_{L} / e=E$ is the external electric field accelerating electron. Therefore the ionization rate in the limit of extremely strong field can be estimated as follows $W_{L} \approx \tau_{i}^{-1}=\omega_{L} a\left[\left(1+I_{i} / m_{e} c^{2}\right)^{2}-1\right]^{-1 / 2}$. Neglecting the relativistic corrections $\left(I_{i} \ll m_{e} c^{2}\right)$ we get linear dependence of the rate on the electric field

$$
W_{L}(E) \approx \omega_{L} a \sqrt{\frac{m_{e} c^{2}}{2 I_{i}}}=\omega_{a} \frac{E}{E_{a}} \sqrt{\frac{I_{H}}{I_{i}}}
$$

Finally, making of use piecewise approach, the formula for the field ionization rate can be extended to the limit of extremely strong field when the potential barrier is strongly suppressed

$$
W_{P W}(E)= \begin{cases}W_{T I}(E), & \text { if } E \leq E_{T I L}, \\ W_{L}(E), & \text { if } E>E_{T I L},\end{cases}
$$

where value $E_{T I L}$ is a threshold electric field and a solution of the transcendent equation $W_{T I}(E)=W_{L}(E)$.

It is interesting to note that according to our calculation the intersection between $W_{T I}(E)$ and $W_{L}(E)$ occurs at reasonable field strength $E_{T I L} \sim E_{c r}$ for all noble gases. For example for all 54 electrons of $\mathrm{Xe}$ $1.15 E_{c r}<E_{T I L}<1.91 E_{c r}$. If the last two electrons $\left(1 s^{1}\right.$ and $1 s^{2}$ electrons) of Xe are excluded then $1.15 E_{c r}<$ $E_{T I L}<1.45 E_{c r}$. The formula proposed in Ref. 28] as a combination of $W_{T I}(E)$ and $W_{Q}(E)$ predicts unphysical value of the threshold electric field $E_{T I Q}=0$ for ionization of $1 s^{2}$ electron of He (see Fig. 2 (a)). It is worth to note that $W_{Q}(E)$ for hydrogen starts to significantly deviate from numerical results at $E=E_{Q L} \simeq 0.4 E_{a}$ where $W_{Q}(E)$ crosses with $W_{L}(E)$ (see Fig. 6 in Ref. [28] and Fig. 2 (b)).

In order to take into account the multiple ionization within one time step of PIC code the MC kinetic numerical model is used [30. The method is based on solution of set coupled first-order differential equations describing evolution of ion charge state [31. The equations can be solved numerically [32, 33] or analytically [30] assuming that the field distribution and the ionization probabilities do not change within the time step. Ionization events are modeled by MC numerical scheme as a random process where the ionization rate is determined by Eq. (5). The energy losses because of ionization are neglected as they are much less than the losses associated with QED cascading (see Sec. IV).

\section{NUMERICAL SIMULATIONS}

Development of laser-assisted QED cascade in noble gases is studied by 3D PIC-MC simulations with code QUILL [11, 34]. The code part based on PIC method models dynamics of a plasma and laser field while the part based on MC method models emission of high energy photons, electron-positron pair creation and field ionization of atoms and ions.

In our simulations two laser pulses propagate towards each other along $x$-axis (see Fig. 3). The laser pulse centers are located in the points $x_{0}=16 \lambda, y_{0}=z_{0}=16.5 \lambda$ and $x_{0}=40 \lambda, y_{0}=z_{0}=16.5 \lambda$ at $t=0$, respectively, 



FIG. 2: (a) The ionization rates $W_{T I}(E)$ (dotted black line 1 ), $W_{P W}(E)$ (solid black line 2$), W_{Q}$ (red line 4) and the rate proposed in Ref. 26] (blue line 3) as functions of the field strength for single-electron ionization of He. (b) The ionization rates $W_{T I}(E)$ (dotted black line 1), $W_{P W}(E)$ (solid black line 2), $W_{Q}$ (red line 4 ) and the rate proposed in Ref. [26] (blue line 3) as functions of the field strength for ionization of hydrogen.

where $\lambda=1 \mu \mathrm{m}$. The pulses are focused on the point $x_{c}=28 \lambda$, which is the center of the gas volume. The laser pulses have linear polarization $\left(E_{z}=B_{y}=0\right)$ and the $y$-component of the electric field at $t=0$ is

$$
\begin{aligned}
E_{y}(\mathbf{r}) & =A(\mathbf{r}) \cos ^{2}\left[\frac{\pi \sqrt{y^{2}+z^{2}}}{2 \sigma(\mathbf{r})}\right] \Upsilon(\mathbf{r}) \\
\Upsilon(\mathbf{r}) & =\cos ^{2} \frac{\pi x}{2 \sigma_{x}} \cos \psi(\mathbf{r})-\frac{\lambda}{4 \sigma_{x}} \sin \psi(\mathbf{r}) \sin \frac{\pi x_{s}}{\sigma_{x}} \\
\psi(\mathbf{r}) & =R(\mathbf{r})-\arctan \frac{d}{x_{R}}-\arctan \frac{x_{s}-d}{x_{R}} \\
R(\mathbf{r}) & =k_{L}\left[x_{s}+\frac{\left(y^{2}+z^{2}\right)\left(x_{s}-d\right)}{2\left(x_{s}-d\right)^{2}+2 x_{R}^{2}}\right] \\
x_{s} & =x-x_{0}, \\
\sigma(\mathbf{r}) & =\frac{\pi \sigma_{0}}{\sqrt{2^{-2} 3 \pi^{2}-4}} \\
\times & {\left[1+\left(\frac{\psi(\mathbf{r})-k_{L} d+\arctan x_{R}^{-1} d}{k_{L} x_{R}}\right)^{2 / 2}\right.} \\
A(\mathbf{r}) & =\frac{a m c \omega_{L}}{e} \frac{\sqrt{x_{R}^{2}+d^{2}}}{\sqrt{x_{R}^{2}+x_{0}^{2}}} \frac{\sigma_{0}}{\sigma(\mathbf{r})} \frac{\pi}{\sqrt{2^{-2} 3 \pi^{2}-4}},
\end{aligned}
$$

where $a=500$ is the laser pulse amplitude, $\sigma_{x}=8 \sqrt{2 \pi} \lambda$ is the pulse length, $d=12 \lambda$ is the distance from the center of the last pulse to gas volume center, $x_{R}=\pi \sigma_{0}^{2} / \lambda$, $\sigma_{0}=3 \lambda, k_{L}=2 \pi / \lambda$. The other components of the electric and magnetic fields at $t=0$ can be calculated from the Maxwell's equations $\nabla \cdot \mathbf{E}=\nabla \cdot \mathbf{B}=0$.

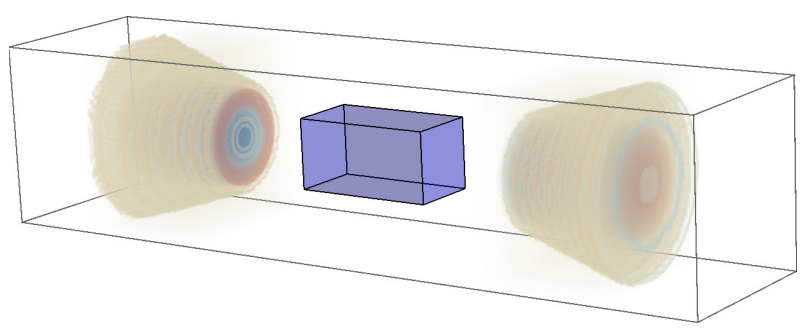

FIG. 3: The scheme of the laser pulse interaction with gas volume. Two counter-propagating laser pulses are focused to the gas volume center.

The gas density is chosen less than $10^{16} \mathrm{~cm}^{-3}$ so that the collisional effects (collisional ionization, bremsstrahlung, pair photoproduction by nuclei etc.) can be neglected (see discussion in Sec. IV). The seed electrons for cascade triggering are produced by the field ionization of the gas atoms. Noble gases He, Ne, Ar, Kr and $\mathrm{Xe}$ are explored. In order to study the contribution of the electrons bound in the different atom shells the densities of the gases is chosen to be reversely proportional to the atomic numbers so that the number of electrons produced after full ionization are the same for all gases. For example, the density of He is $9.03 \times 10^{15} \mathrm{~cm}^{-3}$ in our simulations that is in 27 times higher than the density of Xe, $3.35 \times 10^{14} \mathrm{~cm}^{-3}$. Therefore, in the case of full atom ionization the densities of the ionization-produced electrons for both gases are the same. 
First we study QED cascade development in He. The gas volume in simulation has a length $40 \lambda$ along $x$-axis $(8 \lambda \leq x \leq 48 \lambda)$ and $5 \lambda$ along $y$-axis $(14 \lambda \leq y \leq 19 \lambda)$ and $z$-axis $(14 \lambda \leq z \leq 19 \lambda)$. Further enlargement of the gas volume in all directions does not increase the number of pairs produced in the cascades (see Fig. 4). The gas density is $9.03 \times 10^{15} \mathrm{~cm}^{-3}$.

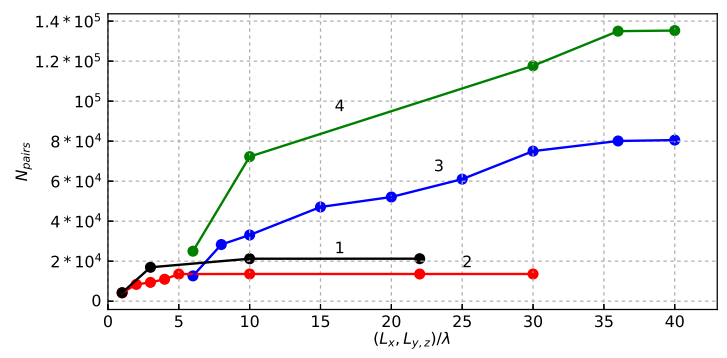

FIG. 4: The number of the pairs produced in the cascade as a function of the gas volume length (along $x$-axis) for He (blue line 1) and for Xe (green line 2) as well as a function of the gas volume width (along $y$ and $z$ axises) for He (red line 3 ) and for Xe (black line 4).

The distributions of the electrons, positrons, ions and $E_{y}$ are shown in Fig. 5 in the different moments of time. The pulse centers cross each other in $x=28 \lambda$ at $t=18 \lambda / c$. The counter-propagating laser pulses generate field structure which is close to the linearly polarized standing wave near $x=28 \lambda$. In the case of He the full ionization of atoms occurs already at the laser pulse front. For $t \geq 10 \lambda / c$ the gas is fully ionized and new electrons are not produced due to field ionization. Most of the produced electrons are pushed out by ponderomotive force of the laser pulse from high intensity region in transverse direction and cannot initiate cascade. The small part of the electrons moves along with the laser pulses thereby forming two counter-propagating relativistic bunches (see Fig. 5(a)). The motion of each bunch are stopped by the counter-propagating laser pulse. Moreover the bunch electrons are trapped in the standing wave nodes corresponding to the minimum of the ponderomotive potential (see Fig. 5(b)) [12, 35, 36].

The typical trajectories of the trapped electrons staying for a long time in region, where laser field peaks $(x=28 \lambda, y=16.5 \lambda, z=16.5 \lambda)$, and the escaping electrons pushed out by the ponderomotive force from high intensity region are shown in Fig. 6. When the wave strength becomes strong enough the bunch electrons start to initiate cascade with prolific pair production. It follows from simulations that the pairs are efficiently produced within time interval $12 \lambda / c \leq t \leq 23 \lambda / c$. The ion density also peaks in the wave nodes because of large uncompensated electron charge accumulated there (see Fig. 5 (d)).

The density of Xe in the simulations is $3.35 \times 10^{14} \mathrm{~cm}^{-3}$ that is in 27 times less than the density of He so that the number of the atomic electrons in the gas volume are
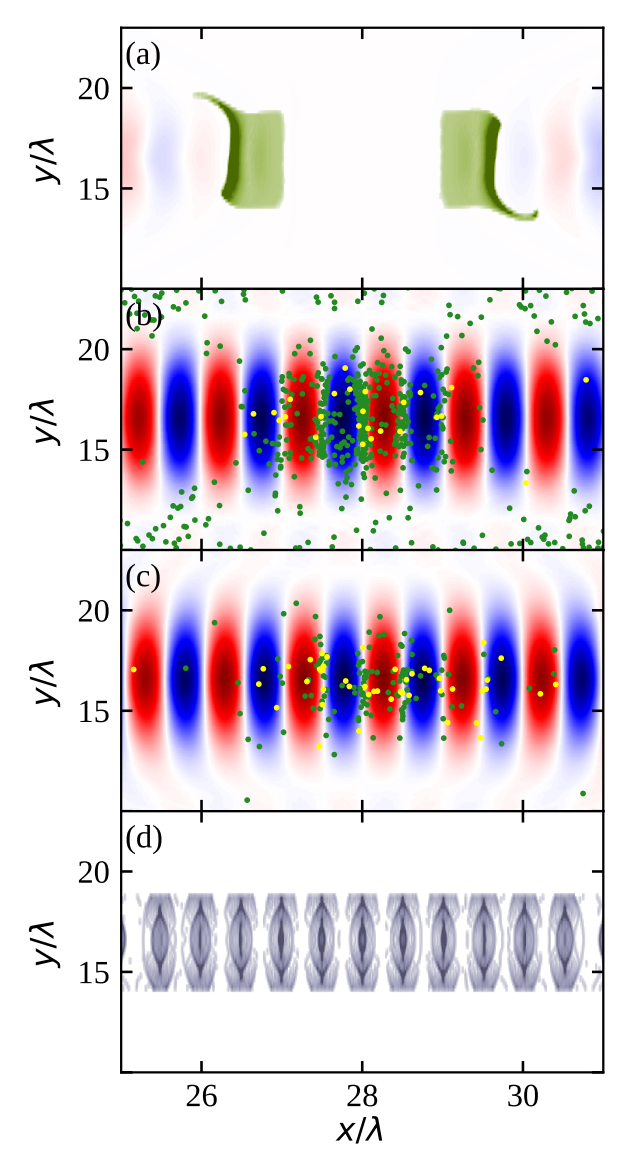

FIG. 5: The distribution of the electrons (green dots), the positrons (yellow dots) and the laser field component $E_{y}$ (blue and red) in $x-y$ plane at $t=8 \lambda / c(\mathrm{a}), t=15 \lambda / c(\mathrm{~b})$ and $t=18 \lambda / c$ (c) for He. The distribution of the He ions in $x-y$ plane at $t=18 \lambda / c(\mathrm{~d})$.

the same for both gases. The cascade development in Xe is shown in Fig. 6. Like for He, the small portion of electrons produced from outer shell of Xe atoms in the laser pulse fronts forms counter-propagating bunches (see Fig. 7(a)). The bunch electrons are trapped in the nodes of the standing wave generated near $x=28 \lambda$, where the laser pulse centers cross each other (see Fig. 7(b)).

In contrast to He the electrons are still produced by field ionization of Xe even after laser pulse crossing $(t \geq$ $18 \lambda / c)$ because the ionization potential of the inner-shell electrons of $\mathrm{Xe}$ atom is in about 3 order of magnitude higher than that of He. Typical trajectories of the $2 s^{1}$ electrons of Xe are shown in Fig. 8. It is seen that some inner-shell electrons escape from the high-intensity region while the other inner-shell electrons undergo oscillations in the strong laser field for a long time and emit highenergy photons. It follows from Fig. 7 that like for $\mathrm{He}$ the density of the electrons, positrons an ions also peak in the nodes of the standing wave forming near volume center.

The dynamics of the inner-shell electron population 


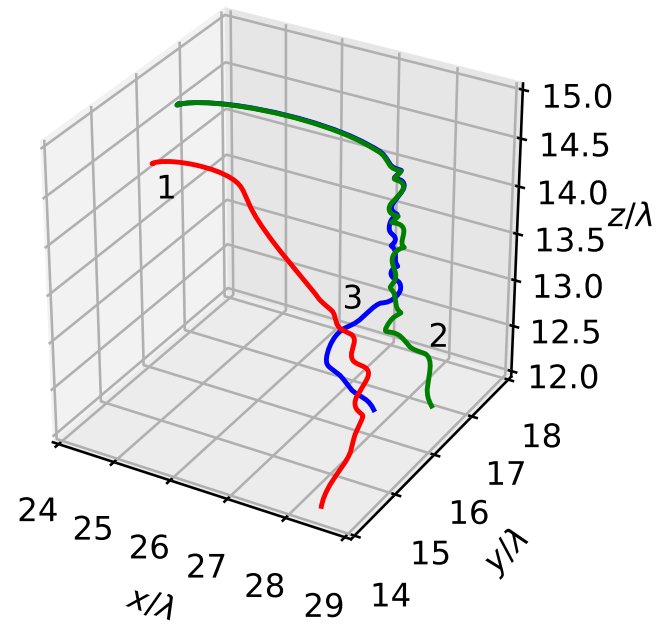

FIG. 6: The trajectory of the escaping electrons pushed out by the ponderomotive force from high intensity region (red line 1) and the trajectory of the trapped electrons staying for a long time in region, where laser field peaks, (blue line 2). The electrons are created by ionization of He atoms.

and of the high-charge ion population is shown in Fig. 9 . It is seen from Fig. 9 that the ions with the highest charges $\mathrm{Xe}^{+52}$ are produced when the laser pulses crosses and the laser field strength peaks. The ionization production rate for the inner-shell electrons and the positron production rate are significant at $7 \lambda / c<t<22 \lambda / c$ when the the laser field is strong. Therefore, the cascade in Xe can be initiated not only by the outer-shell electrons trapped in the standing wave but also by the inner-shell electrons produced when the laser field becomes to be strong enough for cascade development.

The pair numbers as function of time in all noble gases are shown in Fig. 10. The gas densities are normalized to the atomic numbers so that the electron densities in the case of full atom ionization are the same for all gases. The gas densities of $\mathrm{He}, \mathrm{Ne}, \mathrm{Ar}, \mathrm{Kr}$ and Xe are $9.03 \times 10^{15} \mathrm{~cm}^{-3}, 1.81 \times 10^{15} \mathrm{~cm}^{-3}, 10^{15} \mathrm{~cm}^{-3}$, $5.02 \times 10^{14} \mathrm{~cm}^{-3}$ and $3.34 \times 10^{14} \mathrm{~cm}^{-3}$, respectively. The gas volume has a length of $5 \lambda / c$ and a width of $5 \lambda / c$. It is seen from Fig. 4 that ratio $N_{p}(\mathrm{Xe}) / N_{p}(\mathrm{He})$ keeps nearly unchanged with increasing of volume size. Thus, we can expect that the ratio in the positron numbers presented in Fig. 10 for the noble gases will be similar for a macroscopic gas target $(L \gg 40 \lambda / c)$. Despite the fact that Xe density is in 27 times less than He density the number of pairs produced in Xe is in about 2 times larger than that in He. Therefore the inner-shell electrons play an important role in QED cascade triggering. The spectra of the electrons and photons produced in the cascade in He and Xe are shown in Fig. 11. The energy of the particles is slightly higher in Xe than in He.

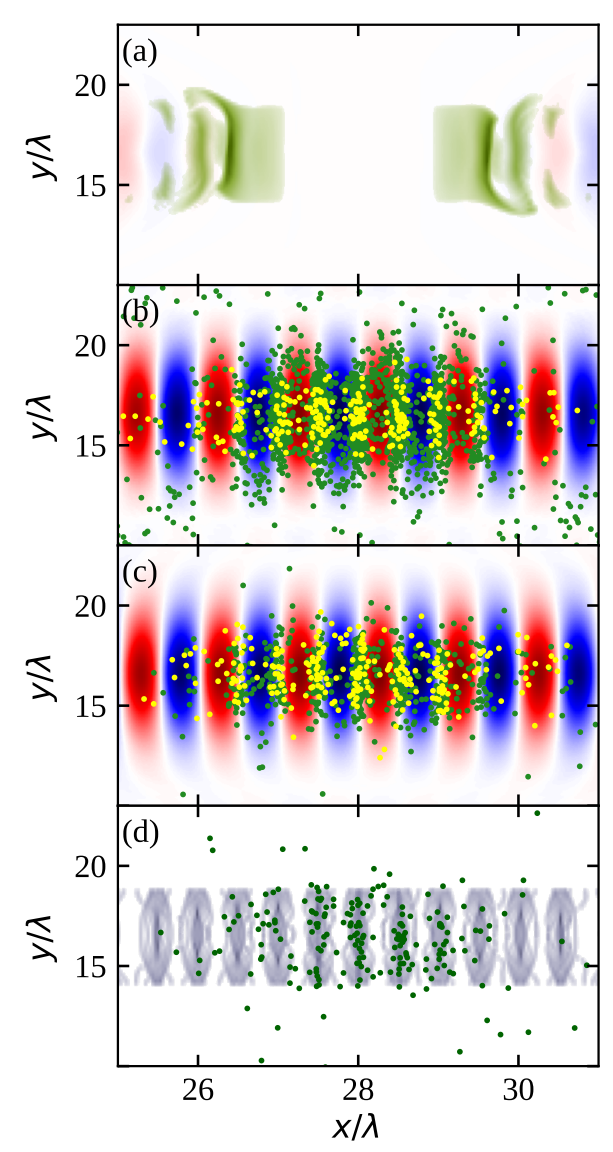

FIG. 7: The distribution of the electrons (green dots), the positrons (yellow dots) and the laser field component $E_{y}$ (blue and red) in $x-y$ plane at $t=8 \lambda / c(\mathrm{a}), t=15 \lambda / c(\mathrm{~b})$ and $t=18 \lambda / c$ (c) for Xe. The distribution of the inner-shell $\left(2 s^{1}\right)$ electrons (green dots) and Xe ions (grey color) in $x-y$ plane at $t=18 \lambda / c(\mathrm{~d})$.

\section{DISCUSSIONS AND CONCLUSIONS}

The simple formula for the field ionization rate covering all range of laser intensity is proposed. The formula based on combination of known expression for tunnel ionization in the low intensity limit [16 18, 23] and the ionization rate formula in the extremely intense limit where the rate is proportional to the strength of the electric field. The linear dependence on the field strength is in a qualitative agreement with numerical TDSE calculations for hydrogen [28] if $E \gg E_{c r}$. However more detailed validation of the proposed formula is needed.

QED cascades in noble gases are studied. It is shown that there are two main mechanisms of seed electron production and cascade initiation in high- $\mathrm{Z}$ gases like Ar, $\mathrm{Kr}$ and Xe: (i) the ionization of the outer-shell electrons moving along with the pulses to the cascade region and (ii) the ionization of the inner-shell electrons created at the instance when the pulses crosses and the total laser 




FIG. 8: The trajectories of the escaping inner-shell $\left(2 s^{1}\right)$ electrons of Xe (line 1) and the inner-shell electrons of Xe (lines 2 and 3 ) staying for a long time in the region, where the laser field peaks.

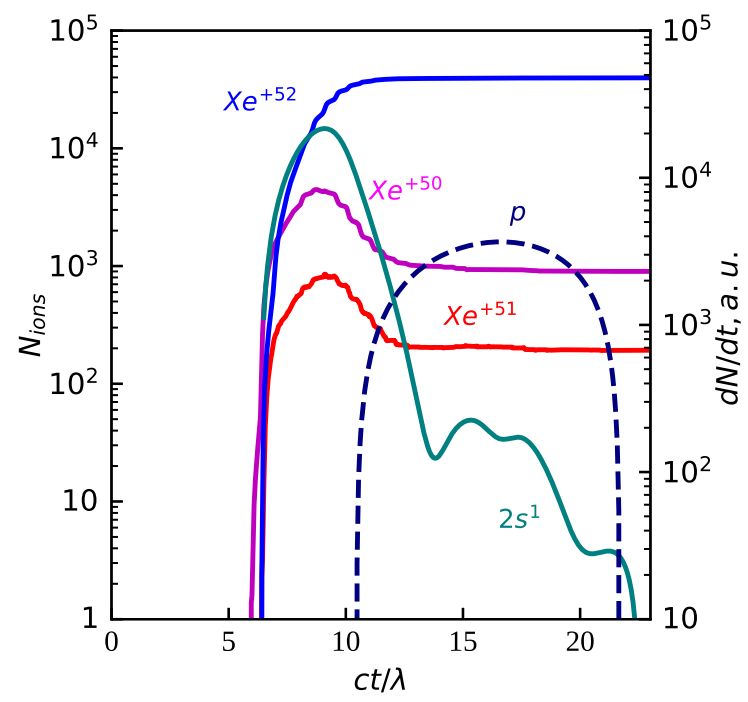

FIG. 9: The number of the high-charge ions, the ionization production rate for the inner-shell electrons and the positron production rate as a function of time in Xe.

field peaks. The ionization potential of $2 s^{1}$ and $2 s^{2}$ electrons of Xe are about $10 \mathrm{keV}$. Those electrons can escape from the ion only at very high field strength. In low-Z gases like $\mathrm{He}$ and $\mathrm{Ne}$ only the first mechanism is possible. These gases are fully ionized in the laser pulse front. Most of the electrons are pushed out by the ponderomotive force of the laser pulse from the high intensity region and cannot initiate cascade. The small part of the pro-

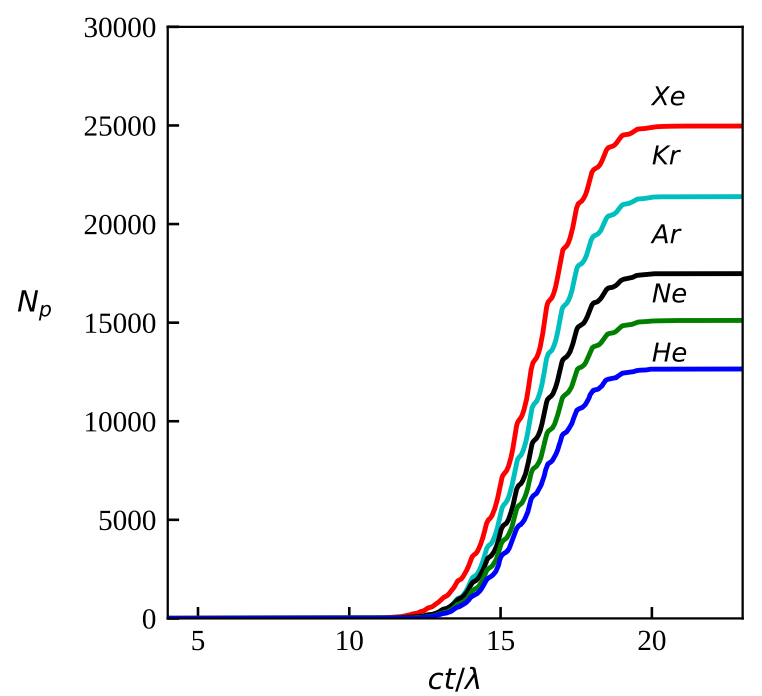

FIG. 10: The pair number as a function of time for $\mathrm{He}, \mathrm{Ne}$, Ar, Kr and Xe.

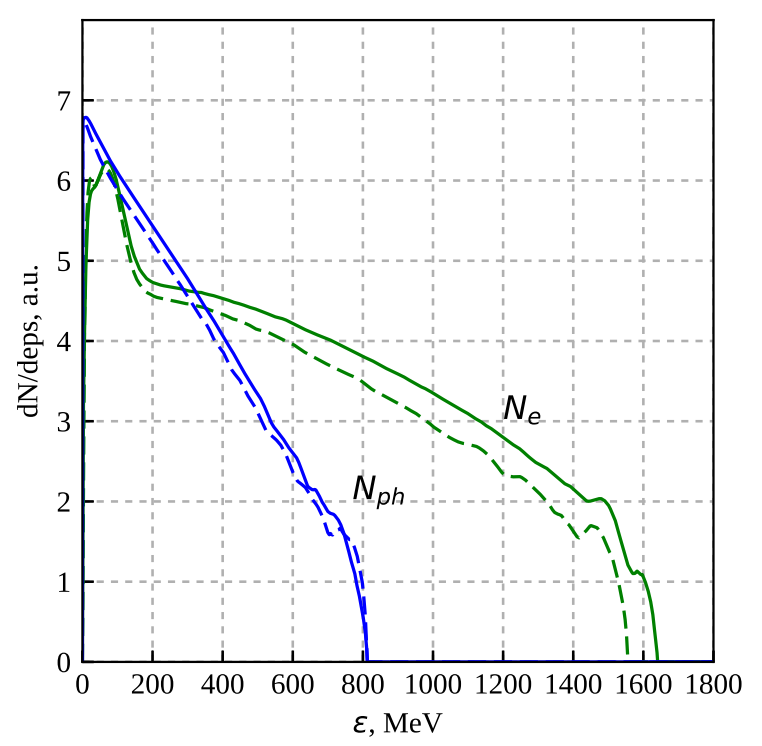

FIG. 11: The spectrum of the photons in He (dashed blue line) and in Xe (solid blue line) and the spectrum of the electrons in $\mathrm{He}$ (dashed green line) and in Xe (solid green line) at $t=$ $28 \lambda / c$.

duced electrons may move along with the laser pulses up to the time instance when the pulses crosses and the laser field becomes strong enough for cascading.

The first mechanism is discussed in Ref. [12 where electron trapping in the cascade region is observed in numerical simulations. It is demonstrated that part of the seed electrons survives up to pulse crossing and is trapped near the electric field nodes of the standing wave formed by the counter-propagating pulses. The second mecha- 
nism are discussed in Ref. [7] where ionization is included in cascade simulations. However the ionization model was too simple and does not take into account probabilistic nature of ionization and sequential multiple ionization of electrons from different shells of high-Z atoms. Moreover all atom electrons leave the atom simultaneously according to this model. We use in our simulations more realistic model providing probabilistic description of field ionization. This model allows us analyse the role of both mechanisms. It follows from our simulations that in high-Z gases like Ar, $\mathrm{Kr}$ and $\mathrm{Xe}$ both mechanisms are important for cascade initialization: the outer-shell electrons are involved in the first mechanism while the innershell electrons are involved in the second one. Comparing pair production in He and Xe (see Figs. 4 and 10 ) we can conclude that the inner-shell electrons of Xe increase pair production in about 2 times for the parameters of interest despite the fact that Xe density is in 27 times less than He density. The result is obtained when the peak laser field strength $2 a=1000$ is close the cascade threshold value. With increasing of the laser intensity the role of the mechanisms can be changed.

QED cascade develops as a result of chain reactions when photon emission caused by electron scattering in the laser field (Compton scattering) alternates with pair photoproduction due to photon scattering in the laser field (Breit-Wheeler process). However high-energy photons and pairs can be also produced by collisional processes neglected in our numerical simulations. Photons can be emitted at electron scattering by ionic or atomic nuclei (bremsstrahlung) while pairs can be created by a high-energy photon interacting with ionic or atomic nuclei. First we estimate the bremsstrahlung contribution to the cascade. The total bremsstrahlung cross-section in the limit $\varepsilon_{e} \gg \varepsilon_{e}^{\prime} \gg m_{e} c_{e}^{2}$ is $\sigma_{b r}=\left(2^{5 / 2} / 3\right) Z^{2} \alpha \cdot r_{e}^{2} \gamma_{e}^{1 / 2}$, where $\varepsilon_{e}$ and $\varepsilon_{e}^{\prime}$ are the electron energies before and after scattering by an ion, respectively, $\gamma_{e}=\varepsilon_{e} /\left(m_{e} c^{2}\right)$ is the relativistic gamma-factor of the electron, $\alpha=e^{2} /(c \hbar) \approx$ $1 / 137$ is the fine structure constant, $r_{e}=e^{2} /\left(m_{e} c^{2}\right) \simeq$ $2.82 \times 10^{-13} \mathrm{~cm}$ is the classic radius of the electron 37. The number of the bremsstrahlung photons can be estimated as follows $N_{b p h} \simeq n_{e} N_{i} c \sigma_{b r} \tau_{c}$, where $n_{e}$ is the density of the relativistic electrons, $N_{i}$ is the number of the the ions in the cascade volume and $\tau_{c}$ is the cascade duration. According to simulation the cascade volume is $V_{c} \sim 5 \lambda \times 5 \lambda \times 5 \lambda$ and the cascade duration is $\tau_{c} \simeq 7 \lambda / c$ (see Fig. 9). For full ionization of xenon gas with $Z=54$ and $n_{g}=3.34 \times 10^{14} \mathrm{~cm}^{-3}$ we obtain $n_{e}=Z n_{g} \simeq 1.8 \times 10^{16} \mathrm{~cm}^{-3}, N_{i}=n_{g} V_{c} \simeq 4.18 \times 10^{4}$ and $N_{b p h} \simeq 9.51 \times 10^{-5}$ where the mean gamma-factor of the electron $\gamma_{e} \simeq 10^{3}$ is used. It follows from estimation that the number of the bremsstrahlung photons is negligible with number of the cascade photons $\left(N_{p h}>10^{5}\right)$.

The number of the electron-positron pairs produced by the high-energy photons near nuclei can be estimated as follows $N_{\text {npair }} \simeq n_{p h} N_{i} c \sigma_{\text {npair }} \tau_{c}$, where $\sigma_{b r} \simeq$ $(28 / 9) Z^{2} \alpha \cdot r_{e}^{2}\left[\ln \left(2 \varepsilon_{p h} / m_{e} c^{2}\right)-(109 / 42)-1.2(\alpha Z)^{2}\right]$ is the cross-section of pair production near nuclei in the relativistic limit $\left(\varepsilon_{e, p h} \gg m_{e} c^{2}\right)$ and $n_{p h}=N_{p h} / V_{c}$ is the photon density in the cascade volume [37. It follows from the simulations that the number of the high-energy photons $\left(\varepsilon_{p h}>1 \mathrm{MeV}\right)$ is less than $N_{p h}<10^{6}$ therefore the number of pairs produced by photons near nuclei is $N_{\text {npair }}<10^{-5}$ that is much less than the pair number produced in the cascade $N_{\text {pair }}>10^{4}$.

The contribution of the collisional ionization can be also estimated by similar way. The cross-section of the collisional ionization for the relativistic electrons is $\sigma_{c i} \simeq$ $\left(2^{7 / 2} \pi^{1 / 2} / 3\right) Z^{2} \alpha \cdot r_{e}^{2} L$, where $L$ is the Coulomb logarithm [38. Even for very large value of $L=20$ the number of electrons produced via collisional ionization $N_{e, c i}=$ $n_{e} N_{i} c \sigma_{c i} \tau_{c} \simeq 0.016$ is much less than the number of the electrons created via field ionization $N_{e} \gg N_{i}>10^{4}$ in the cascade volume. Thus collision ionization can be neglected in the cascade modeling.

The electrons can be also produced by the collision of high-energy photons with atoms or partially ionized ions (photoelectric effect). This effect of one-photon ionization is not included in our numerical scheme for field ionization. The photoelectric cross-section peaks for photons with energy $\varepsilon_{p h}<m_{e} c^{2}$ and does not exceed $\sigma_{\text {phe }}<10^{-19} \mathrm{~cm}^{-2}$ [39]. It follows from Fig. 11 that the mean energy of the cascade photons is more than $100 \mathrm{MeV}$ and the photons with energy $\varepsilon_{p h}<m_{e} c^{2}$ belong the low-energy part of the photon spectra. The number of the photons radiated by the electron moving in the laser field per unit time can be estimated as follows $d N_{p h} / d \varepsilon_{p h}=\varepsilon_{p h}^{-1} d I / d \varepsilon_{p h}$ and $d N_{p h} / d \varepsilon_{p h} \simeq \omega_{L}^{-1} 0.021 \alpha a_{S} \chi_{e}^{2 / 3} \gamma_{e}^{-4 / 3}\left(\varepsilon_{p h} / m_{e} c^{2}\right)^{-2 / 3}$, where the approximation for the low-energy part of the synchrotron radiation spectrum is used, $d I / d \varepsilon_{p h}$ is the synchrotron radiation spectrum 40 and $a_{S}=$ $m_{e} c^{2} / \hbar \omega_{L}$. The number of the low-energy photons emitted during cascade development is $N_{p h}\left(\varepsilon_{p h}<m_{e} c^{2}\right) \simeq$ $0.063 \alpha a_{S} \chi_{e}^{2 / 3} \gamma_{e}^{-4 / 3}\left(\omega_{L} \tau_{c}\right) \simeq 4$, where $\chi_{e} \simeq 10$ is taken from the simulations. The number of the electron produced via photoelectric effect in the cascade region during cascade development is $N_{e, p h e}=\left(N_{p h} / V_{c}\right) N_{i} c \sigma_{p h e} \tau_{c} \simeq$ $10^{-7}$ that is much less than the number of the electrons created via field ionization $N_{e} \gg N_{i}>10^{4}$. Therefore photoelectric effect can be also neglected in the cascade modeling. The losses associated with the ionization are also neglected in the simulations because the ionization energy $\left(<0.1 m_{e} c^{2}\right)$ is in several order of magnitude less than the mean electron energy in the laser field $\left(\sim 10^{3} m_{e} c^{2}\right)$.

Finally it is demonstrated the Xe among noble gases is more appropriate to facilitate QED cascading. However the additional effects like the laser pulse propagation from the focusing parabolas to the cascade volume, the accurate description of the gas target and ionization dynamics should be taken into account for realistic simulations of possible laboratory experiments. 


\section{Acknowledgments}

This work was supported by part by the "Basis" Foundation Grant No. 17-11-101-1. The numerical simula- tions of QED cascades was supported by the Russian Science Foundation Grant No. 16-12-10383.
[1] A. R. Bell and J. G. Kirk, Phys. Rev. Lett. 101200403 (2008).

[2] A. Di Piazza, C. MÂžller, K. Z. Hatsagortsyan, C. H. Keitel, Rev. Mod. Phys. 84, 1177 (2012).

[3] N. B. Narozhnyi, A. M.. Fedotov, Phys. Usp. 58, 95 (2015).

[4] N. P. Zafir, Eur. Phys. J. Special Topics 223, 1221 (2014).

[5] J. P. Zou, C. Le Blanc, D. N. Papadopoulos, G. Cheriaux, P. Georges, G. Mennerat, F. Druon, L. Lecherbourg, A. Pellegrina, P. Ramirez, F. Giambruno, A. Freneaux, F. Leconte, D. Badarau, J. M. Boudenne, D. Fournet, T. Valloton, J. L. Paillard, J. L. Veray, M. Pina, P. Monot, J. P. Chambaret, P. Martin, F. Mathieu, P. Audebert, and F. Amiranoff, High Power Laser Science and Engineering 3, e2 (2015).

[6] E. G. Gelfer, A. A. Mironov, A. M. Fedotov, V. F. Bashmakov, E. N. Nerush, I. Yu. Kostyukov, N. B. Narozhny, Physical Review A 92, 022113 (2015).

[7] M. Tamburini, A. Di Piazza, C. H. Keitel, Sci. Rep. 7, 5694 (2017).

[8] A. Gonoskov, A. Bashinov, S. Bastrakov, E. Efimenko, A. Ilderton, A. Kim, M. Marklund, I. Meyerov, A. Muraviev, A. Sergeev, Ultra-bright $\mathrm{GeV}$ photon source via controlled electromagnetic cascades in laser-dipole waves, arXiv: 1610.06404

[9] G. Breit, J. A. Wheeler, Phys. Rev. 46, 1087 (1934).

[10] A. M. Fedotov, N. B. Narozhny, G. Mourou, G. Korn, Phys. Rev. Lett. 105, 080402 (2010).

[11] E. N. Nerush, I. Yu. Kostyukov, A. M. Fedotov, N. B. Narozhny, N. V. Elkina, and H. Ruhl, Phys. Rev. Lett. 106, 035001 (2011).

[12] M. Jirka, O. Klimo, S. V. Bulanov, T. Zh. Esirkepov, E. Gelfer, S. S. Bulanov, S. Weber, and G. Korn, Phys. Rev. E 93, 023207 (2016).

[13] I. Gonoskov, A. Aiello, S. Heugel, G. Leuchs, Phys. Rev. A 86, 053836 (2012).

[14] E. N. Nerush, I. Yu. Kostyukov, Nuclear Instruments and Methods in Physics Research A 653, 7 (2011).

[15] A. A. Mironov, A. M. Fedotov, N. B. Narozhnyi, Quantum Electronics 46, 305 (2016).

[16] A. M. Perelomov, V. S. Popov, M. T. Terent'ev, Sov. Phys. JETP 23, 924 (1966).

[17] V. S. Popov, Phys. Usp. 47, 855 (2015).

[18] B. M. Karnakov, V. D. Mur, S. V. Popruzhenko, V. S. Popov, Phys. Usp. 58, 3 (2015).
[19] I. I. Artemenko, A. A. Golovanov, I. Yu. Kostyukov, T. M. Kukushkina, V. S. Lebedev, E. N. Nerush, A. S. Samsonov, D. A. Serebryakov, JETP Letters 104, 883 (2016).

[20] L. V. Keldysh, Sov. Phys. JETP 20, 1307 (1965).

[21] F. A. Ilkov, J. E. Decker, S. L. Chin, Phys. B: At. Mol. Opt. Phys. 25, 405 (1992).

[22] Handbook of Mathematical Functions, edited by M. Abramowitz and I. A. Stegun (Dover, New York, 1972).

[23] M. V. Ammosov, N. B. Delone, V. P. Krainov, Sov. Phys. JETP 641191 (1986).

[24] N. B. Delone, V. P. Krainov, Physics-Uspekhi 42 669, (1999).

[25] D. Bauer, Phys. Rev. A 55, 2180 (1997).

[26] X. M. Tong and C. D. Lin, J. Phys. B 38, 2593 (2005).

[27] V. P. Krainov, J. Opt. Soc. Am. B, 14, 425 (1997).

[28] D. Bauer and P. Mulser, Phys. Rev. A 59, 569 (1999).

[29] Q. Zhang, P. Lan, P. Lu, Phys. Rev. A 90, 043410 (2014).

[30] R. Nuter, L. Gremillet, E. Lefebvre, A. Levy, T. Ceccotti, and P. Martin, Phys. Plasmas 18, 033107 (2011).

[31] S. C. Rae and K. Burnett, Phys. Rev. A 46, 1084 (1992).

[32] M. Chen, E. Cormier-Michel, C. G. R. Geddes, D. L. Bruhwiler, L. L. Yu, E. Esarey, C. B. Schroeder, W. P. Leemans, J. Comp. Phys. 236220 (2013).

[33] A. V. Korzhimanov, E. S. Efimenko, A. V. Kim, and S. V. Golubev, Quantum Electron. 43, 217 (2013).

[34] E. N. Nerush and I. Yu. Kostyukov, Probl. Atom. Sci. Tech. 4, 3 (2010).

[35] G. Lehmann, and K. H. Spatschek, Phys. Rev. E 85, 056412 (2012).

[36] A. Gonoskov, A. Bashinov, I. Gonoskov, C. Harvey, A. Ilderton, A. Kim, M. Marklund, G. Mourou, and A. Sergeev, Phys. Rev. Lett. 113, 014801 (2014).

[37] V. B. Berestetskii, E. M. Lifshits, and L. P. Pitaevskii, Quantum Electrodynamics (Pergamon Press, New York, 1982).

[38] D. Wu, X. T. He, W. Yu, S. Fritzsche, Particle-in-cell simulations of laser-plasma interactions at solid densities and relativistic intensities: the role of atomic processes, arXiv preprint arXiv:1703.05127.

[39] E. Storm, H. I. Israel, Nucl. Data Tables A7, 565 (1970).

[40] V. N. Baier, V. M. Katkov, and V. M. Strakhovenko, Electromagnetic Processes at High Energies in Oriented Single Crystals (Singapore, World Scientific 1998). 